\title{
Principal components analysis for Hindi digits recognition
}

\begin{abstract}
The recognition process depends on the how features are extracted. There are several ways for feature extraction but the most important is to extract the most effective features and can distinct between patterns. In this research, an approach is proposed to recognize Hindi numerals. Initially image is enhanced and normalized. After that, PCA is applied for feature extraction. Recognition is performed by using first and second Norm. Another two more norms were proposed named ENorm and EEuclidean. Results showed 93.5\%, 94.79\%, 95\% and $94.79 \%$ recognition accuracy when applying first norm, ENorm, second norm and EEuclidean respectively.
\end{abstract}

